\title{
Universal Screening for SARS-CoV-2 in Asymptomatic Pregnant Women: A Multi-center Experience
}

\author{
Shreyasi Sharma ${ }^{1} \mathbb{D} \cdot$ Sanjay Wazir ${ }^{2} \cdot$ Rajagopal Kishore Kumar $^{3}$
}

Received: 8 October 2020 / Accepted: 11 March 2021 / Published online: 30 April 2021

(c) Federation of Obstetric \& Gynecological Societies of India 2021

\begin{abstract}
Background Novel coronavirus (SARS-CoV-2) is responsible for the current global pandemic and understandably, Obstetrics is not spared. Private maternity hospitals have a unique challenge of reassuring unaffected patients of uneventful delivery with the lowest possible rate of coronavirus infection while consequently offering compassionate and state of art services to women who turn out to be positive for SARS-CoV-2. This has led to a routine SARS-CoV-2 testing of all patients before admission in many of the private hospitals in India. The current study was undertaken to determine the incidence of SARSCOV-2 among asymptomatic pregnant women and to ascertain the utility of universal screening in these women.

Methodology A retrospective observational multi-center study was conducted over a period of approximately 5 months (1-May-2020 to 10-September-2020) in a chain of privately run maternity hospitals with presence in multiple cities across India. All asymptomatic pregnant women were tested for SARS-CoV-2 prior to elective/emergency hospital admission.

Results Among 4158 women tested, 54 (1.3\%) were positive for SARS-CoV-2 and intra partum and postnatal period was uneventful for all of them.

Conclusion Universal screening should be continued as preferred approach to ensure low anxiety levels of delivering women and safety of frontline workers. Further, universal screening helps avoid emergence of maternity centers as virus clusters by effective isolation of identified positive cases and minimizing points of contact.
\end{abstract}

Keywords SARS-CoV-2 $\cdot$ Universal screening $\cdot$ SARS-CoV-2 incidence $\cdot$ Asymptomatic pregnant women

It has been over 10 months since the new strain of coronavirus (SARS-CoV-2) responsible for the current pandemic has been documented in Wuhan City, China [1]. Although the fatality is low, its high rate of infectivity has led to the disruption of normal life globally. Overburdening of health care facilities, scare among patients and healthcare workers are

Shreyasi Sharma, Senior Consultant Fetal Medicine; Sanjay Wazir, Senior Consultant Paediatrics and Neonatology; Rajagopal Kishore Kumar, Senior Consultant Paediatrics and Neonatology.

Shreyasi Sharma

shreyasi2003@gmail.com

1 Department of Fetal Medicine, Cloudnine Hospital Gurugram, Sector 47, Gurgaon 122018, India

2 Department of Paediatrics and Neonatology, Cloudnine Hospital - Gurugram, Gurgaon, India

3 Department of Paediatrics and Neonatology, Cloudnine Hospital - Bengaluru, Bengaluru, India a few of the multitude of problems faced by the health care system at present. Understandably, Obstetrics is not spared.

Private maternity hospitals have a unique challenge of reassuring unaffected patients of uneventful delivery with the lowest possible rate of infections while consequently offering compassionate care to all those diagnosed with SARS-CoV-2. This has led to a routine SARS-CoV-2 testing of all patients before admission. Thus, the current study was undertaken to determine the incidence of SARS-CoV-2 among asymptomatic pregnant women and to examine the universal screening paradigm for these women.

A retrospective observational multi-center study was conducted over a period of approximately 5 months (1-May2020 to 10 -September-2020) in a chain of privately run maternity hospitals having presence in multiple cities across India. All asymptomatic pregnant women (4158 women across Bengaluru, Chennai, Mumbai, Pune and Gurugram) who were planned for delivery/cesarean section were tested prior to admission. A nasopharyngeal swab was obtained and testing was performed using real-time RT-PCR (reverse 


\section{Geographical distribution of study}

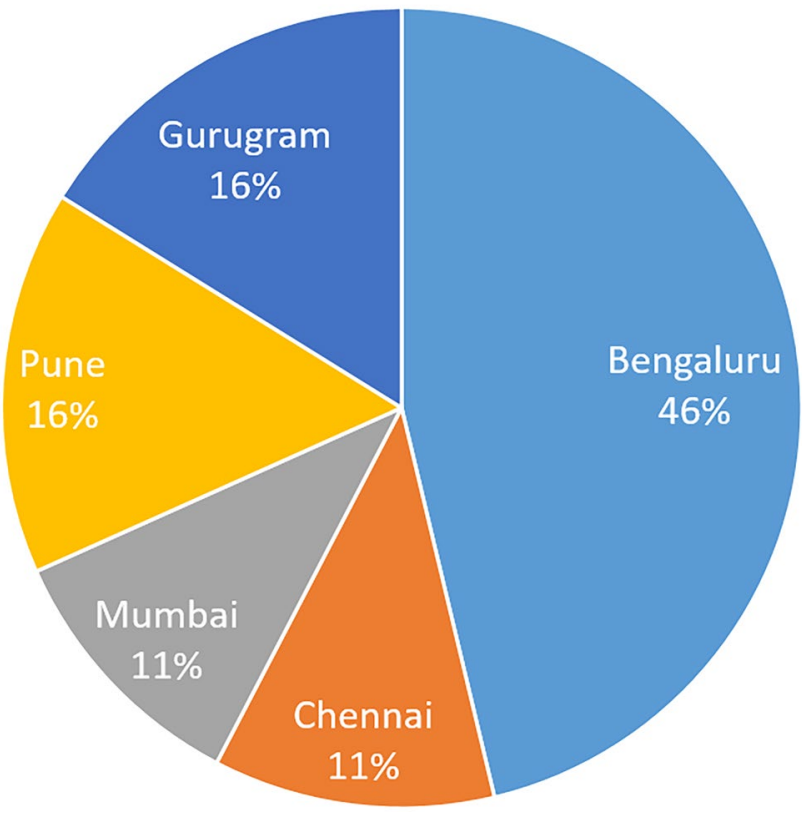

Fig. 1 Geographical distribution of the study cohort

transcription polymerase chain reaction). If tested positive, the woman was delivered in the designated area for COVID positive patients.

Of the 4158 women, 54 (1.3\%) tested positive for SARS$\mathrm{CoV}-2$. None of these women developed any symptoms of SARS-CoV-2 during the entire duration of hospitalization and intra partum and postnatal period was uneventful for all of them. Geographical distribution of the study population is provided in Fig. 1 and geography-wise incidence levels are provided in Fig. 2. Also, to account for higher sample concentration from Bengaluru skewing the incidence levels, we normalized the incidence percentage for getting the composite incidence level using district-level population as collected during 2011 National Census exercise. The population normalized SARS-CoV-2 incidence among asymptomatic pregnant women is $\sim 1.4 \%$. Further, among the cohort of women who tested negative for SARS-CoV-2, none developed any symptoms during the course of hospitalization.
Thus, retesting in these women was not required as per hospital protocol.

Considering the scale of pandemic and lack of guidelines, universal screening seems to be a logical solution despite additional cost. The reasons for this assertion are multifold. For the patients, especially pregnant women, anxiety is compounded when they anticipate admission to a health care facility. A part of it can be assigned to the fear of contracting the disease from apparently asymptomatic individuals. For the hospital, information about SARS-CoV-2 status of the patient can aid in planning hospital isolation practices, bed assignments, neonatal care and use of personal protective equipment (PPE) [2]. Sutton et al. support the same, however $13.7 \%$ of the asymptomatic cohort tested positive for SARS-CoV-2 in their study [2]. In contrast, in our study, only $1.3 \%$ of such women tested positive for SARS-CoV-2. Despite the fact that the data are derived from the metropolitan regions of both countries, strict enforcement of lockdown strategies in India during the period of study and higher awareness and availability of designated COVID-hospitals in these regions might be responsible for our findings.

Further, none of the women who tested positive for SARS-CoV-2 developed any symptoms during the hospital stay. This is similar to the findings of Sutton et al., where only $1(0.03 \%)$ patient developed febrile illness that was presumed to be due to SARS-CoV-2 [2]. This highlights that it is extremely unlikely for such women to develop severe symptoms. Thus, the utility of screening might only be limited to reduce transmission than guiding the course of management in these women.

Universal screening of asymptomatic pregnant women prior to admission seems to be a double-edged sword as in the process of tapping into the possible benefits, it in itself overburdens the laboratories for an extremely low diagnostic yield. Despite the drawbacks, universal screening should be continued as preferred approach to reduce heightened anxiety levels of delivering women due to the pandemic. Universal screening also ensures the safety of frontline workers and helps avoid emergence of maternity centers as virus clusters by effective isolation of SARS$\mathrm{CoV}-2$ positive patinets and minimizing points of contact. 


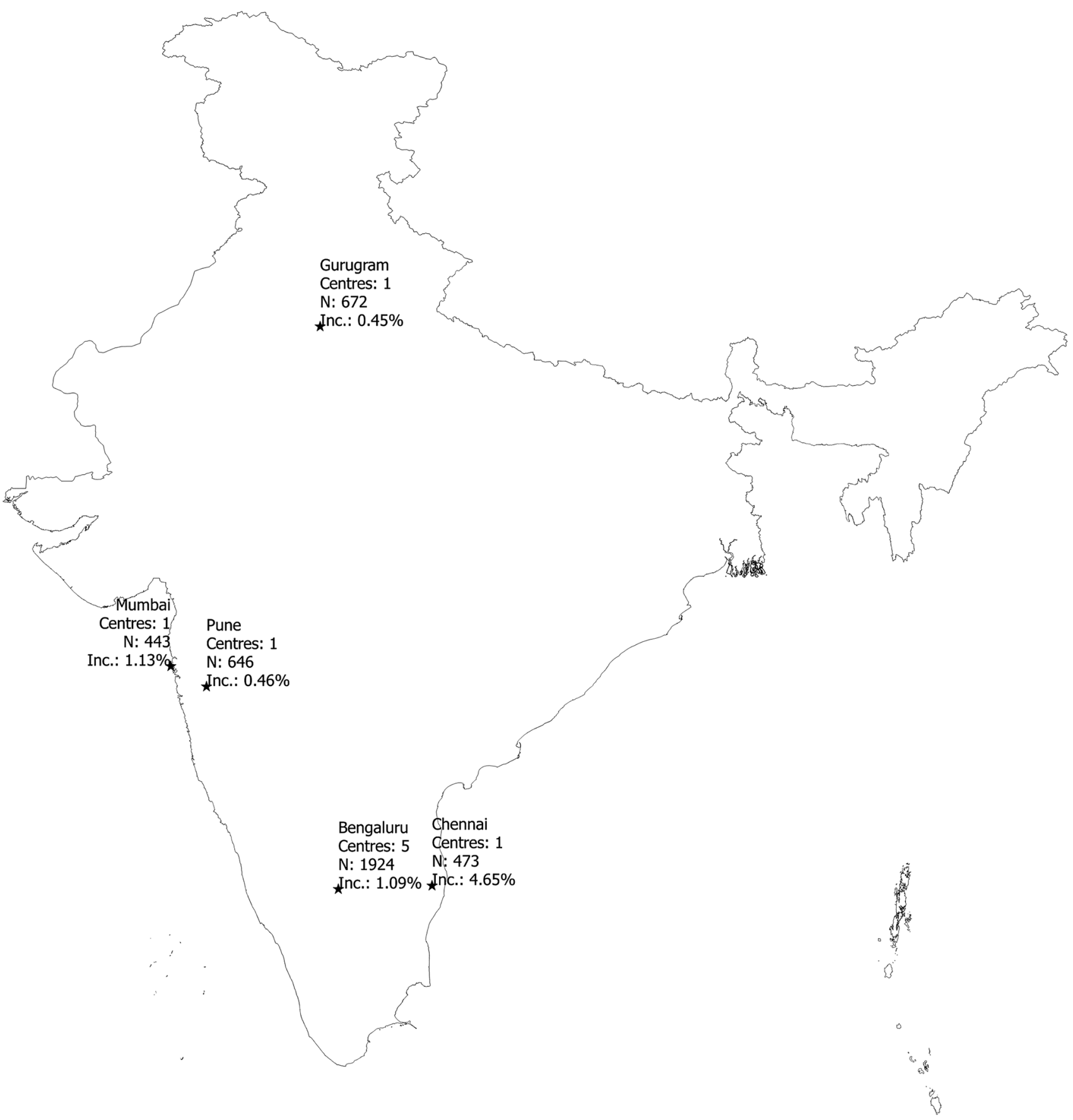

Fig. 2 Geography-wise incidence of SARS-CoV-2 in asymptomatic pregnant women

Although this is first such study in India and an attempt was made to capture data from diverse geographical locations, the small population cohort remains a limitation. Further studies are necessary to address this key issue. 


\section{Compliance with ethical standards}

Conflicts of interest The authors declare no conflict of interest.

Ethical Approval Due permissions from concerned authorities were obtained before collecting data. Strict confidentiality of patient data was maintained.

\section{References}

1. Team NCPERE. Vital surveillances: the epidemiological characteristics of an outbreak of 2019 novel coronavirus diseases (COVID-19)—China. China CDC Weekly. 2020;8:113-22.

2. Sutton D, Fuchs K, D'Alton M, Goffman D. Universal screening for SARS-CoV-2 in women admitted for delivery. N Engl J Med. 2020;382(22):2163-4.

Publisher's Note Springer Nature remains neutral with regard to jurisdictional claims in published maps and institutional affiliations.

\section{About the Author}

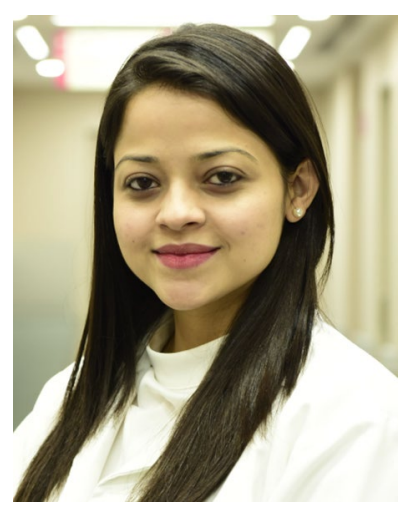

Shreyasi Sharma is a multiple award-winning Fetal Medicine Specialist, currently working as Senior Consultant, Fetal Medicine at Cloudnine Hospital, Gurgaon. She completed her MD in Obstetrics and Gynecology from PGIMER, Chandigarh, MBBS from Madras Medical College, Chennai and holds a fellowship in Fetal Medicine. She is Fetal Medicine Foundation (FMF UK) certified for obstetric scanning and fetal interventions. Her areas of interest include screening for preterm labor, fetal growth restriction, Rh-isoimmunisation, diagnostic and therapeutic fetal procedures and clinical genetics. 